Hiroyasu Iwasa $\cdot$ Masahiko Kurabayashi $\cdot$ Ryozo Nagai

Yusuke Nakamura • Toshihiro Tanaka

\title{
Genetic variations in five genes involved in the excitement of cardiomyocytes
}

Received: May 22, 2001 / Accepted: June 8, 2001

\begin{abstract}
We provide here 29 genetic variations, including 28 novel ones, in five genes that are potentially involved in the excitement of cardiomyocytes: we found 4 in KCNA10, 2 in $K C N K 1,8$ in KCNK6, 11 in SLC18A1 (VMAT1), and 4 in $S L C 6 A 2$ (norepinephrine transporter). We also examined their allelic frequencies in a Japanese population of long QT syndrome-affected and nonaffected individuals. These data would be useful for genetic association studies designed to investigate acquired arrhythmias.
\end{abstract}

Key words Long QT syndrome · Single-nucleotide polymorphism - Japanese population - Norepinephrine transporter - VMAT1 - Two-pore domain potassium channel . KCNA10

\section{Introduction}

Long QT syndrome (LQTS), an arrhythmogenic disorder characterized by prolongation of the QT interval on electrocardiograms (ECGs), often causes syncope or sudden cardiac death as a result of recurrent and lethal arrhythmia. Five genes in which inherited mutations are responsible for this syndrome have been identified to date: KCNA9

H. Iwasa $\cdot$ Y. Nakamura $\cdot$ T. Tanaka $(\square)$

Laboratory of Molecular Medicine, Human Genome Center,

Institute of Medical Science, University of Tokyo, 4-6-1

Shirokanedai, Minato-ku, Tokyo 108-8639, Japan

Tel. +81-3-5449-5375; Fax +81-3-5449-5406

e-mail: toshitan@ims.u-tokyo.ac.jp

H. Iwasa $\cdot$ M. Kurabayashi

Second Department of Internal Medicine, Gunma University School of Medicine, Gunma, Japan

R. Nagai

Department of Cardiovascular Medicine, Graduate School of

Medicine, University of Tokyo, Tokyo, Japan
(KVLQT1, KCNQ1), KCNH2 (HERG), KCNE1, KCNE2, and SCN5A (Bennett et al. 1995; Curran et al. 1995; Wang et al. 1996a,b; Splawski et al. 1997; Abbott et al. 1999). A mutant form in at least one of these five genes can be detected in nearly $40 \%$ of LQTS families, but the abnormalities responsible for the syndrome in the remaining families seem to be in other, unknown genes.

Potassium channels are the most heterogenous group of ion channels in terms of structure and function. Some are voltage-gated channels, and most LQTS-associated genes [KCNA9 (KVLQT1), KCNH2 (HERG), KCNE1, KCNE2] are voltage-gated potassium-channel genes. KCNA10, the most recently cloned voltage-gated potassium-channel gene, may potentially participate in the cardiac action potential (Lang et al. 2000). The most recently discovered class of potassium channel is the two-pore-domain potassium-channel family, although the physiological function of this class in cardiac myocytes has not yet been clarified. TWIK-1 (encoded by KCNK1), TASK-1 (encoded by $K C N K 3$ ), and TWIK-2 (encoded by KCNK6) are potential candidates to generate a potassium current in the cardiac action potential.

As sympathetic denervation of the left heart can sometimes prevent sudden cardiac death of LQTS patients (Bhandari et al. 1984), molecules that might potentially play parts in sympathetic activity are also candidates. Heterozygous deficiency of $S L C 18 A 2$, the gene encoding vesicular monoamine transporter (VMAT2), can induce a prolonged QT interval and sudden death in knockout mice (Itokawa et al. 1999). Information on variations among individuals in genes expressed in the monoaminergic system can provide better knowledge of arrhythmogenic conditions, and so other monoaminnergic genes, SLC18A1, encoding vesicular monoamine transporter 1 (VMAT1) (Weiche et al. 1994), and SLC6A2, encoding norepinephrine transporter, are also candidate genes linked to arrhythmogenic disorders (Shannon et al. 2000).

Here we report multiple single-nucleotide polymorphisms (SNPs) and other genetic variations among five candidate genes for LQTS along with their allelic frequencies among normal and LQTS-affected Japanese subjects. 
Table 1. PCR primers used to cover the coding region of $K C N A 10, K C N K 1, K C N K 3, K C N K 6, S L C 18 A 1$, and $S L C 6 A 2$

\begin{tabular}{|c|c|c|c|c|c|}
\hline Gene & Exon $^{a}$ & Forward primer & Reverse primer & $\begin{array}{l}\text { Annealing } \\
\text { temperature }\end{array}$ & $\begin{array}{l}\text { GenBank } \\
\text { accession } \\
\text { number }\end{array}$ \\
\hline \multirow[t]{2}{*}{ KCNA10 } & 1.1 & AGG CTG AGG ATC TTC ATG C & GAA GGT GAA CCA CAC GAT G & 62 & NM_005549 \\
\hline & 1.2 & TGG TCT CGG TGT TGG TTG & GAG AAG AGA GAC AGG ATG GAC & 62 & NM_005549 \\
\hline \multirow[t]{9}{*}{ KCNK1 } & 1.1 & AAA CAT CGC CCG AGA GAG C & GCG CAC GCA CGA GCT G & 62 & NM_002245 \\
\hline & 1.2 & GCG GCG GTG GAG AAG AT & CGA GGA GAA GAC CAC TGC G & 62 & NM_002245 \\
\hline & 1.3 & TTC CTG GTG CTG GGC TAC & GAC ACG CCG TAG TTG CTG & 62 & NM_002245 \\
\hline & 1.4 & AGC AGC AGC TGG AGC AGT & TGT GGG TTG TGG GCG AG & 64 & NM_002245 \\
\hline & 2.1 & CGC CTC AGT GAC CTT GTT CTC & AGA AGC CCC AGC GGA TGT & 62 & NM_002245 \\
\hline & 2.2 & GTG GTC CAG CGC ATC AC & GAT TCC AGG AAG TTC CAG TC & 62 & NM_002245 \\
\hline & 2.3 & CCG CTG TCT TCT CAG TCC TG & AAT AAA GGA GAC AGA AGG AGC AG & 62 & NM_002245 \\
\hline & 3.1 & ATG TTG AGA TCA CAC TAA GAC AGT & GGA GAA GGA CAG TTG GTC A & 62 & NM_002245 \\
\hline & 3.2 & TCA GGT GCA CAT CAT AGA GC & TGA CCC TGG TGC TCT AGC & 58 & NM_002245 \\
\hline \multirow[t]{9}{*}{ KCNK3 } & 1.1 & GGT GGT GCT GAA GGG ACA & GAA GGT GCA CAC GAT GAG C & 62 & AF065163 \\
\hline & 1.2 & ATG AAG CGG CAG AAC GTG & CGC TCC AGC TCC TCG TAG & 62 & AF065163 \\
\hline & 1.3 & GCG CGC TAC AAC CTC AG & GAC GAC TCC CGG AGC CC & 62 & AF065163 \\
\hline & 2 & СТT TTT СТС СТС TTT CCC ACT & GTT GGC CAT GGA CAC GTC & 60 & AF065163 \\
\hline & intron2 & AAG AAG GGG CTG GGC AT & CAG TGC TCG TAG TGG GAG AAG & 60 & AF065163 \\
\hline & 3.1 & ACT GTT TAC ACT CGT GCA TCA GC & ATG ACC GTG AGG CCC GTA & 62 & AF065163 \\
\hline & 3.2 & GCC TTC AGC TTC GTC TAC ATC & GTA GAC GTT GCG GAA GCC & 62 & AF065163 \\
\hline & 3.3 & CTC ATC CAC GGC GGC AG & GCG ACG AGT GGC TCT GCT & 62 & AF065163 \\
\hline & 3.4 & GAC CTC TCC ACG TCC GAC AC & GCC CCC AGG TGC TCC AG & 62 & AF065163 \\
\hline \multirow[t]{4}{*}{ KCNK6 } & 1 & CTG GGT CTG GGA GCA CTG & CCC CGA GCA GAG CAG ATA & 58 & AF117708 \\
\hline & 2 & AGA GCC TGC ACC CTT CAT & ATC CAA ATG TCA CCT TCT CAG & 60 & AF281303 \\
\hline & 3 & GCT ACT TAG GAG GAG GCT GAG & GCC CAG GAA GAG GTA GAC TG & 62 & AF117708 \\
\hline & 4 & CAC TGC TGC TGA CTC ACG T & ATC ACC TGC TGT GCT CGT G & 62 & AF117708 \\
\hline \multirow[t]{18}{*}{ SLC18A1 } & 1 & GCA ATT AAA TCT TTG TGG ACA & GAC ACA AAT CAC AAG GAA AGA T & 58 & NM_003053 \\
\hline & 2.1 & AAA TAT CAT CCT ACT ACT TGC A & CAG AAT GGT CCG GAG CA & 55 & NM_003053 \\
\hline & 2.2 & CCC CAG TCC GGC CAT CA & ACA GCA AAT TAA CCC TCA GCA & 58 & NM_003053 \\
\hline & 3.1 & CCA TCA CCT GTC CTC TTT GAC & GAT GGT GCT GGC AGT GTC A & 62 & NM_003053 \\
\hline & 3.2 & TAG CAT GGA TGA ATG ACA CTG & TAC TCC TGT GTA CCC TGC G & 58 & NM_003053 \\
\hline & 4 & GTG CCT GTT GGA ATT GTA TCT & GAT AAA ATC AAA GGT AAT CAG TGA & 58 & NM_003053 \\
\hline & 5 & AGT TAT CTT GCT ATT CTC TCT GC & CAT GTA AAT ATA GGA TCT GAG GTA & 58 & NM_003053 \\
\hline & 6 & GGG CCA GCT GCA TCT GTA & ACA CCT CGG GAA CAC CTG & 58 & NM_003053 \\
\hline & 7 & ACC CTC ACC TCT GGA ATC TG & ACC TGG GCT GTC ACC TGC & 55 & NM_003053 \\
\hline & 8 & CCA GCT AAT CAG TAG ACA CGC & TGC ATT CAG CCA TCC TGT AT & 58 & NM_003053 \\
\hline & 9 & TAT GCA CTG TAC AAA TGG ACA T & GGG AGA CTG TTC TGT GAG CA & 58 & NM_003053 \\
\hline & 10 & CGG CTG CTC ACA GAA CAG T & CAT TGA ACG AAA GGA CTC ATG & 58 & NM_003053 \\
\hline & 11 & TAC GAG ACA AGG CAT TTT AGA & СTC TTC CAT CTT ACA TTT CTA CTG & 58 & NM_003053 \\
\hline & 12 & GAC CCA TCT GGA GTC CTG AT & TTG TTT TCT TTG AGG GCA CT & 58 & NM_003053 \\
\hline & 13 & GAC CCA TCT GGA GTC CTG AT & TTG TTT TCT TTG AGG GCA CT & 58 & NM_003053 \\
\hline & 14 & CTG ACT GTG TCC TGT TTG CTC & AAA GAG TAG ACA GGG GAA AGT G & 58 & NM_003053 \\
\hline & 15 & ATA GAC ACT TAT GAC TTT GAT GTA T & CTC TTT TAA CAG TCG GTG CT & 58 & NM_003053 \\
\hline & 16 & AAA AAT GAT AAT AAT GTG CAG TG & GGT CAC TGA GGC ATC ATG A & 58 & NM_003053 \\
\hline \multirow[t]{18}{*}{ SLC6A2 } & 1.1 & AAA GTT CCT CTC GCC AGC & CAG CAG GCA CTG GAC GC & 60 & X91117 \\
\hline & 1.2 & GGT GAA GGA GCG CAA C & CAA ATT CCC AGC CCG AC & 58 & X91117 \\
\hline & 2 & ACT CTG CCC CTG TGT CCT C & CCT TTA TGA ACA ACC GTG ACT & 60 & $\mathrm{X} 91120$ \\
\hline & 3.1 & СТС СТА ССТ ТАС ССС СТG TC & GCT TGG GGT CGG TAC AGT & 62 & X91121 \\
\hline & 3.2 & CTG CCC TGG ACC GAC TG & CAA GCA CTG GCC TCA ACA & 62 & X91121 \\
\hline & 4 & ССС TCC ССТ СТС CTC TG & TAA GTG AAA GAG AAA CAC AGA GAT & 60 & X91122 \\
\hline & 5 & CAC CTG AAC TTA TCC ATT GC & CAA GGC TTG GTG GTC ACT & 60 & X91123 \\
\hline & 6 & TGA TGG CTT TTG TCT GCT G & CCC CAC AAG AGT CAA TCC & 60 & X91124 \\
\hline & 7 & GGG ACT TGA CCT CAC TGT GC & CTC AGC TCC CGC CTC AGT & 62 & X91125 \\
\hline & 8 & CCA TTG ATG AGG TCC TTG CTG T & CAG TGG GAT GGG GGA TAG GTA T & 58 & X91126 \\
\hline & 9 & GAG ACC CTA ATT CCT GCA C & ATT GAA ATG CGG CCT CAG & 62 & X91127 \\
\hline & 10 & AAT CAG TTC CCA CGT TTG AC & GGA GGA CTG GGA GCT GAG & 62 & X91127 \\
\hline & 11 & GGC CTG CCC TGT GTG TGC & CCC CCT CCC CAC ATG CAG & 65 & X91118 \\
\hline & 12.1 & СТT ТСТ СТС ССТ ТСТ СТG C & GCT GAG GAA CTT ATA GAT GAC G & 58 & X91118 \\
\hline & 12.2 & ATC GCC CTG TCC TCC ATG CC & GTC TTC CCC CTC AGC & 62 & X91118 \\
\hline & 13 & GCA GGA TCA AAT AGC AGG TG & CCA GGG GTC TAG GCT TCA C & 62 & X91119 \\
\hline & 14.1 & TCT GTC CCC ACC ATG TCA TC & AAG AGA GGA TCA GAA AGG ACA ACT & 65 & X91119 \\
\hline & 14.2 & CCC ACC TCG GAC ACC ATC & AAG TCA TTT TCC TGT TTG CTC & 62 & X91119 \\
\hline
\end{tabular}

PCR, polymerase chain reaction

${ }^{a}$ Exons followed by dots and sequential numbers were subdivided for analysis 
Table 2. Genetic variations of five candidate genes for long QT syndrome

\begin{tabular}{|c|c|c|c|c|c|c|c|}
\hline \multirow[b]{2}{*}{ Gene } & \multirow[b]{2}{*}{ Nucleotide change } & \multirow[b]{2}{*}{ Amino acid change } & \multirow[b]{2}{*}{ Regions } & \multicolumn{2}{|c|}{$\begin{array}{l}\text { Frequency of } \\
\text { minor allele }\end{array}$} & \multicolumn{2}{|c|}{$\begin{array}{l}\text { Number of } \\
\text { chromosomes } \\
\text { examined }\end{array}$} \\
\hline & & & & LQTS & control & LQTS & control \\
\hline \multirow[t]{4}{*}{ KCNA10 } & $348 \mathrm{G}>\mathrm{A}$ & E116E & Exon1 & 0.36 & 0.35 & 160 & 188 \\
\hline & $399 \mathrm{G}>\mathrm{T}$ & R133R & Exon1 & 0.11 & 0.09 & 160 & 100 \\
\hline & $658 \mathrm{G}>\mathrm{A}$ & V330M & Exon1 & 0.08 & 0.07 & 160 & 100 \\
\hline & $741 \mathrm{G}>\mathrm{A}$ & L247L & Exon1 & 0.33 & 0.30 & 160 & 100 \\
\hline \multirow[t]{2}{*}{$K C N K 1$} & $\mathrm{nt}(-17)($ ins12) & & $5^{\prime}$ UTR & 0.48 & 0.47 & 160 & 100 \\
\hline & $600 \mathrm{C}>\mathrm{T}$ & A200A & Exon2 & 0.005 & 0.01 & 160 & 100 \\
\hline \multirow[t]{8}{*}{ KCNK6 } & $-144 \mathrm{G}$ & & $5^{\prime}$ UTR & 0.01 & 0.03 & 160 & 190 \\
\hline & $-63 \mathrm{G}>\mathrm{T}$ & & $5^{\prime}$ UTR & 0.005 & Rare $^{\mathrm{b}}$ & 160 & 190 \\
\hline & $294 \mathrm{C}>\mathrm{T}$ & F98F & Exon1 & 0.005 & Rare $^{\mathrm{b}}$ & 160 & 190 \\
\hline & $449 \mathrm{C}>\mathrm{T}$ & $\mathrm{T} 150 \mathrm{I}$ & Exon3 & 0.02 & 0.04 & 160 & 500 \\
\hline & $576 \mathrm{~T}>\mathrm{C}$ & F192F & Exon3 & 0.06 & 0.08 & 160 & 190 \\
\hline & $718+9 \mathrm{G}>\mathrm{C}$ & Intronic variant & Intron 3 & 0.005 & Rare $^{\mathrm{b}}$ & 160 & 190 \\
\hline & $719-10 \mathrm{G}>\mathrm{A}$ & Intronic variant & Intron3 & 0.005 & Rare $^{b}$ & 160 & 190 \\
\hline & $775 \mathrm{G}>\mathrm{A}$ & V259M & Exon4 & 0.09 & 0.08 & 160 & 190 \\
\hline \multirow[t]{11}{*}{ SLC18A1 } & $10 \mathrm{~A}>\mathrm{C}$ & $\mathrm{T} 4 \mathrm{P}$ & Exon2 & 0.37 & 0.37 & 160 & 100 \\
\hline & $31 \mathrm{C}>\mathrm{T}$ & R11W & Exon2 & 0.005 & 0.006 & 160 & 194 \\
\hline & $293 \mathrm{G}>\mathrm{C}$ & S98T & Exon3 & 0.29 & 0.28 & 160 & 180 \\
\hline & $407 \mathrm{~T}>\mathrm{C}$ & T136I & Exon3 & 0.30 & 0.27 & 160 & 194 \\
\hline & $447 \mathrm{G}>\mathrm{A}$ & M149I & Exon3 & 0.005 & 0.005 & 160 & 180 \\
\hline & 489-14(insA) & Intronic variant & Intron4 & 0.09 & 0.09 & 160 & 192 \\
\hline & $809 \mathrm{~A}>\mathrm{T}$ & D270L & Exon7 & 0.005 & 0.005 & 160 & 196 \\
\hline & $815 \mathrm{G}>\mathrm{C}$ & A272P & Exon7 & 0.005 & 0.005 & 160 & 196 \\
\hline & $814+21 \mathrm{~A}>\mathrm{C}$ & Intronic variant & Intron7 & 0.38 & 0.36 & 160 & 180 \\
\hline & $1065 \mathrm{C}>\mathrm{T}$ & L355L & Exon11 & 0.09 & 0.07 & 160 & 192 \\
\hline & $1094+13 \mathrm{G}>\mathrm{A}$ & Intronic variant & Intron 11 & 0.08 & 0.06 & 160 & 190 \\
\hline \multirow[t]{4}{*}{ SLC6A2 } & $362 \mathrm{G}>\mathrm{A}$ & A121Q & Exon2 & 0.005 & 0.005 & 160 & 190 \\
\hline & $1022+6 \mathrm{~A}>\mathrm{T}$ & Intronic variant & Intron6 & 0.005 & Rare $^{b}$ & 160 & 188 \\
\hline & $1287 \mathrm{G}>\mathrm{A}$ & $\mathrm{T} 429 \mathrm{~T}$ & Exon9 & 0.26 & 0.26 & 160 & 100 \\
\hline & $1590+23 \mathrm{~T}>\mathrm{C}$ & Intronic variant & Intron11 & 0.03 & 0.04 & 160 & 188 \\
\hline
\end{tabular}

5'UTR, 5' untranslated region; LQTS, long QT syndrome

${ }^{a}$ Nucleotide numbering starts from the ATG start codon

${ }^{\mathrm{b}}$ Rare indicates that it was not identified in normal controls

\section{Materials and methods}

In the course of a search for mutations in these candidate genes in Japanese LQTS patients, we identified 29 SNPs that were present in the genomic DNA of probands of 80 independent LQTS-affected families, but in which no mutation had been detected in genes responsible for LQTS. Our screening method was described previously (Itoh et al. 1998). In brief, we prepared genomic DNA from blood samples according to standard protocols after obtaining written informed consent from each participant. All exons of each gene being scrutinized, as well as flanking intronic sequences, were amplified by newly designed polymerase chain reaction (PCR) primers to cover the coding region of each gene (Table 1) and analyzed by single-strand conformation polymorphism (SSCP) analysis. Aberrant conformers were directly sequenced with an ABI 3700 DNA Analyzer (Applied Biosystems, Foster City, CA, USA). In the two genes most recently screened (KCNK6 and $K C N A 10$ ), all LQTS-affected samples were screened by direct sequencing analysis.

To investigate the allelic frequencies of each polymorphism in our control population, we distinguished allelespecific sequences either by using PCR-RFLP (restriction fragment length polymorphism) techniques or by hybridizing allele-specific oligonucleotides to DNA from normal, unrelated individuals in the manner described by Saiki et al. (1986).

\section{Results and discussion}

In total, we confirmed 27 SNPs ( 4 in KCNA10, 1 in KCNK1, 8 in KCNK6, 4 in SLC6A2, 10 in SLC18A1) and two insertion/deletion polymorphisms, and we examined the frequency of each allele in the Japanese population as summarized in Table 2. Of the two insertion/deletion polymorphisms, one was a single-nucleotide insertion/deletion in SLC18A1 and the other was an insertion/deletion of 12 nucleotides, TTTGGCTTTGGC, in the $5^{\prime}$ untranslated region (5'UTR) of KCNK1. We found no polymorphism in coding regions or flanking sequences of KCNK3. These polymorphisms have not been reported previously, except for the $1287 \mathrm{G}>$ A polymorphism in SLC6A2 (Stober et al. 1996). The frequency of each polymorphism showed no significant difference between LQTS and control samples.

Some nonsynonymous polymorphisms (R11W, D270L, and A272P in SLC18A1, and R121Q in SLC6A2) were each 
detected in only one of the probands of independent LQTSaffected families. However, since these variations did not cosegregate with the disease, we consider that they did not cause LQTS. We also found some silent SNPs and intronic variants in one of the LQTS-affected samples, but none of them seemed to lead to splicing abnormalities. Hence, at present we consider these five substitutions to be rare polymorphisms.

We believe that the data reported here will provide useful information for association studies designed to identify genes related to nonfamilial arrhythmias, for instance, druginduced arrhythmia.

Acknowledgments This work was supported in part by a Grant-in-Aid from the Ministry of Education, Science, Sports, and Culture of Japan. We are grateful to Kaori Sato for secretarial assistance.

\section{References}

Abbott GW, Sesti F, Splawski I, Buck ME, Lehmann MH, Timothy KW, Keating MT, Goldstein SA (1999) MiRP1 forms $\mathrm{I}_{\mathrm{Kr}}$ potassium channels with $H E R G$ and is associated with cardiac arrhythmia. Cell 97:175-187

Bennett PB, Yazawa K, Makita N, George AL Jr (1995) Molecular mechanism for an inherited cardiac arrhythmia. Nature 376:683-685

Bhandari AK, Scheinman MM, Morady F, Svinarich J, Mason J, Winkle R (1984) Efficacy of left cardiac sympathectomy in the treatment of patients with the long QT syndrome. Circulation 70:10181023

Curran ME, Splawski I, Timothy KW, Vincent GM, Green ED, Keating MT (1995) Molecular basis for cardiac arrhythmia: HERG mutations cause long QT syndrome. Cell 80:795-803
Itoh T, Tanaka T, Nagai R, Kikuchi K, Ogawa S, Okada S, Yamagata S, Yano K, Yazaki Y, Nakamura Y (1998) Genomic organization and mutational analysis of $K V L Q T 1$, a gene responsible for familial long QT syndrome. Hum Genet 103:290-294

Itokawa K, Sora I, Schindler CW, Itokawa M, Takahashi N, Uhl GR (1999) Heterozygous VMAT2 knockout mice display prolonged QT intervals: possible contributions to sudden death. Brain Res Mol Brain Res 71:354-357

Lang R, Lee G, Liu W, Tian S, Rafi H, Orias M, Segal AS, Desir GV (2000) KCNA10: a novel ion channel functionally related to both voltage-gated pottasium and CNG cation channels. Am J Physiol Renal Physiol 2278:F1013-F1021

Saiki RK, Bugawan TL, Horn GT, Mullis KB, Erlich HA (1986) Analysis of enzymatically amplified beta-globin and HLA-DQ alpha DNA with allele-specific oligonucleotide probes. Nature 324:163166

Shannon JR, Flattem NL, Jordan J, Jacob G, Black BK, Biaggioni I, Blakely RD, Robertson D (2000) Orthostatic intolerance and tachycardia associated with norepinephrine-transporter deficiency. $\mathrm{N}$ Engl J Med 342:541-549

Splawski I, Tristani-Firouzi M, Lehmann MH, Sanguinetti MC, Keating MT (1997) Mutations in the hminK gene cause long QT syndrome and suppress $\mathrm{I}_{\mathrm{Ks}}$ function. Nat Genet 17:338-340

Stober G, Nothen MM, Porzgen P, Bruss M, Bonisch H, Knapp M, Beckmann H, Propping P (1996) Systematic search for variation in the human norepinephrine transporter gene: identification of five naturally occurring missense mutations and study of association with major psychiatric disorders. Am J Med Genet 67:523-532

Wang Q, Li Z, Shen J, Keating MT (1996a) Genomic organization of the human SCN5A gene encoding the cardiac sodium channel. Genomics 34:9-16

Wang Q, Curran ME, Splawski I, Burn TC, Millholland JM, VanRaay TJ, Shen J, Timothy KW, Vincent GM, de Jager T, Schwartz PJ, Towbin JA, Moss AJ, Atkinson DL, Landes GM, Connors TD, Keating MT (1996b) Positional cloning of a novel potassium channel gene: KVLQT1 mutations cause cardiac arrhythmias. Nat Genet $12: 17-23$

Weiche E, Schafer MK, Erickson JD, Eiden LE (1994) Localization of vesicular monoamine transporter isoforms (VMAT1 and VMAT2) to endocrine cells and neurons in rat. J Mol Neurosci 5:149-164 